\title{
TÉCNICAS Y PROCEDIMIENTOS APLICABLES A LA AUDITORÍA ACADÉMICA
}

\author{
Carlos Alfonso Egúsquiza Pereda \\ Docente-Facultad de Ciencias Contables
}

\begin{abstract}
RESUMEN
Los resultados de la presente investigación serán aplicados por los contadores públicos, otros profesionales y sociedades, asesoras y consultoras, dedicados a las actividades de auditoría en la evaluación del proceso de enseñanza-aprendizaje y gestión empresarial, para asegurar la calidad de los servicios de la auditoría académica, incluyendo a las universidades, organismos de supervisión y control, como la Contraloría General de la República, el Ministerio de Educación, las universidades, instituciones superiores y centros educativos en general.

Las técnicas y procedimientos de auditoría tradicionales devienen a través del tiempo en desuso o son poco aplicables a la naturaleza y objetivos de la auditoría académica. Por ello la presente investigación cientifica ha estudiado y evaluado las técnicas y procedimientos más eficaces y de bajo costo en cuanto a su aplicación, para asegurar la calidad de los servicios de auditoría académica. Siendo el objetivo central de estos: la obtención de evidencias competentes, suficientes e irrefutables.
\end{abstract}

Estas técnicas y procedimientos estarán encaminados a la evaluación del proceso enseñanza-aprendizaje, gestión, control y evaluación de resultados de la educación en los distintos niveles. En la presente investigacion se plantean las siguientes técnicas y procedimientos aplicables a la auditoría académica:

- Recolección de la información y evidencias de auditoría:

- Entrevistas.

- Encuestas.

- Cuestionarios.

- Estudios y opinión de expertos.

- Análisis y estudio académico:

- Análisis de costo-beneficio y costo de efectividad.

- Evaluación del sistema de organización y gestión.

- Estudio de mercado-necesidades de profesionales y técnicos y ámbito laboral.

- Evaluación de la capacidad instalada, los niveles de producción y los costos ociosos.

- Evaluación de bibliotecas, laboratorio y sistemas comunicación e información.

- Estudio de las políticas académicas.

- Lectura de las actas de consejos, comités, asambleas generales, asambleas universitarias, etc.

- Estudio de la legislación aplicable a la educación.

- Estudio o evaluación de los planes curriculares y sílabos.

- Evaluación de los procesos de enseñanza-aprendizaje.

- Análisis de los flujogramas de los procesos de enseñanza-aprendizaje.

- Estudio y evaluación del reglamento sobre concurso, ratificación y promoción de docentes.

- Estudio de las bases y procedimientos seguidos en el concurso de docentes.

- Evaluación de los expedientes de los docentes: concurso, ratificación y promoción. 
- Evaluación de los informes de la Dirección de la Escuela Académico Profesional con respecto al semestre o año académico.

- Revisión de las variables y criterios utilizados en la evaluación del desempeño académico del docente.

- Estudio de las fichas de opinión de los alumnos con respecto al desempeño del docente.

El presente estudio tiene un impacto técnico-profesional, social, institucional y empresarial. Los beneficios y bondades serán aprovechados por los contadores públicos, profesionales en general, dedicados a la actividad de auditoría académica, a las universidades, a los colegios profesionales, docentes universitarios, estudiantes, empresas, firmas de asesores y consultores, y autoridades de las instituciones educativas en general.

Palabras clave: Auditoría académica hacia la excelencia educativa.

\section{INTRODUCCIÓN}

Teniendo en cuenta que la AUDITORÍA ACADÉMICA se ubica dentro de la Auditoría Integrada o Integral, de acuerdo al enfoque que subrayamos en la investigación sobre enfoques y objetivos de la auditoría integrada; el tema, materia de la presente investigación, se halla en el campo de la auditoría integral.

En la investigación anterior, tratamos sobre la auditoría académica, esta vez, siendo la auditoría académica una disciplina dirigida al estudio o evaluación de la gestión académica, que comprendeno solo lo relativo a la acción gerencial o gestión puramente dicha, orientada a la evaluación del "corazón del negocio", sino lo relativo al proceso enseñanza-aprendizaje de empresas o instituciones educativas. En tal sentido, sibien es cierto que las técnicas de investigación utilizadas por la auditoría tradicional contable- que aplica las técnicas de investigación empleadas por las ciencias exactas o puras, siendo la Auditoría una disciplina que corresponde al campo de las ciencias sociales o aplicadas, las técnicas y procedimientos generalmente conocidas, tales como: observación, inspección, indagación, muestreo, análisis, circularización y confirmación de saldos, arqueos, investigación, declaración, cálculos y sumas, reconciliación bancaria,etc., y usadas por la auditoría contable, no van a ser tan eficaces en la obtención de evidencias de auditoría, o de forzarse su uso, algunas de ellas pueden ser onerosas en su aplicación, en cuanto al tiempo y costo, por ende no pueden ser de mucha relevancia o eficacia.

Tiene también como antecedentes, las exigencias del mundo globalizado y competitivo del siglo XXI: Los avances económicos cultural, científico $y$ tecnológico, hacen que los profesionales dedicados a la auditoría estemos inmersos en la per manente y continua investigación, explorando nuevas técnicas y métodos de estudio y analizando los problemas de eficiencia empresarial o institucional, bajo la inspiración de las organizaciones aprehendientes e inteligentes en la era de los conocimientos de la infor mación.

Esta investigación se ha desarrollado aplicando los siguientes métodos y técnicas de investigación científica:

- Recopilación bibliográfica.

- Recolección de la información.

- Encuestas y entrevistas.

- Determinación del tamaño de la nuestra.

- Análisis de la información. 
- Redacción del informe final.

- Publicación del informe.

\section{PLANTEAMIENTO DEL ESTUDIO}

En el Perú, Latinoamérica y, porque no decirlo, a nivel mundial, no existen trabajos o investigaciones que orienten la búsqueda apropiada de evidencias de auditoría competentes, suficientes e irrefutables aplicados a la auditoría académica, nos referimos a las técnicas y procedimientos aplicables en materia de la evaluación de la actuación de los directivos, funcionarios y profesores de las instituciones o empresas académicas: promotores, rectores, decanos, directores de Escuelas académico profesionales, coordinadores académicos, directores en general, consejeros de universidades, institutos superiores y centros educativos, en general; en cuanto se refiere a la evaluación del "corazón del negocio" de las empresas o instituciones educativas para asegurar la producción de profesionales y técnicos de alta calidad para el mundo competitivo y globalizado.

El estudio se ha orientado a ubicar y seleccionar nuevas técnicas y procedimientos aplicables a la auditoría académica para que sirvan como instrumento o herramienta de los profesionales, de las sociedades asesoras y consultoras dedicadas a la actividad de evaluación, supervisión y control de centros educativos en general, en todos los niveles: Inicial, primarıa, secundaria y superior.

\section{DESCRIPCIÓN DEL ESTUDIO}

\section{Antecedentes}

Los antecedentes del presente estudio los encontramos en los últimos eventos internacionales de la profesión del contador público, XXIII y XXIV Conferencia Interamericana de Contabilidad de 1999 y 2001 de San Juan de Puerto Rico y Punta del Este, Uruguay, respectivamente, en los que sean han planteado los nuevos horizontes de la auditoría moderna y de avanzada, a la par con la globalización económica, científica y tecnológica, las disciplinas de las ciencias empresariales, en cuanto se refieren a la gestión, supervisión y control de las empresas e instituciones cada vez se hacen más complejas y disímiles; por lo tanto, requieren que los problemas se resuelvan con la participación de equipos multidisciplinarios de profesionales $y$ técnicos; por ello, la auditoría tradicional o financiera se hace cada vez menos trascendente e importante, ya que hoy en día se exige la evaluación o la auditoría referida al estudio del "corazón de las empresas o instituciones", lo que trata indudablemente la auditoría integral, en la que halla ubicada la auditoría académica. En la investigación anterior tratamos al respecto, esta vez la investigación está dirigida a las técnicas y procedimientos aplicables a la auditoría académica.

\section{HIPÓTESIS}

En la investigación indicada se han planteado las siguientes hipótesis o instrumentos de investigación científica:

$\mathrm{H}_{1}$ : La auditoría Académica no tiene técnicas y procedimientos definidos para suaplicaciónodesarrolloen nuestromedio. $\mathrm{H}_{2}$ : Las técnicas y procedimientos de auditoría tradicional requieren evaluación y estudio para relacionar con la auditoría académica.

$\mathbf{H}_{3}$ : Las empresas o, instituciones educativas o académicas no están informadas de la importancia y 
trascendencia de la auditoría académica, de la misma manera los profesionales y las empresas consultoras dedicadas a la actividad de auditoría no cuentan con técnicas y procedimientos definidos ni una metodología adecuada para llevar a cabo una auditoría académica.

\section{TÉCNICAS Y PROCEDIMIENTOS APLICABLES}

Las técnicas y procedimientos para la evaluación del proceso enseñanzaaprendizaje, gestión, control y evaluación de resultados de la educación en los distintos niveles de la educación, son las siguientes:

1. Recolección de la información y evidencias de auditoría:

- Entrevistas.

- Encuestas.

- Cuestionarios.

- Estudios y opinión de expertos.

2. Análisis y estudio académico:

- Análisis de costo-beneficio y costo de efectividad.

- Evaluación del sistema organización y gestión.

- Estudio de mercado - necesidades de profesionales y técnicos y a su ámbito laboral.

- Evaluación de la capacidad instalada, los niveles de producción y los costos ociosos.

- Evaluación debibliotecas, laboratorio y sistemas de comunicación e información.

3. Estudio de las políticas académicas.

4. Lectura de las actas de consejos, comités, asambleas generales, asambleas universitarias, etc.

5. Estudio de la legislación aplicable a la educación.
6. Estudio o evaluación de los planes curriculares y sílabos.

7. Evaluación de los procesos de enseñanza-aprendizaje.

8. Análisis de flujogramas de los procesos de enseñanza-aprendizaje.

9. Estudio y evaluación del reglamento sobre concurso, ratificación y promoción de docentes.

10. Estudio de las bases y procedimientos seguidos en el concurso de docentes.

11. Evaluación de los expedientes de los docentes: concurso, ratificación y promoción.

12. Evaluación de los informes de la Dirección de la Escuela Académico Profesional con respecto al semestre o año académico.

13. Revisión de las variables y criterios utilizados en la evaluación del desempeño académico del docente.

14. Estudio de las fichas de opinión de los alumnos con respecto al desempeño del docente.

\section{CONCLUSIONES}

1. Las técnicas y procedimientos aplicables a la auditoría académica que están dirigidas a la evaluación de la gestión académica de las empresas o instituciones educativas o académicas, en los distintos momentos de la curva de la vida económica de organizaciones aprehendientes e inteligentes bajo la filosofía de calidad total y excelencia, dirigidosal "corazón del negocio", tienen que estar relacionadas en cada caso, de acuerdo a las características y peculiaridades de la empresa o instituciones, "las técnicas de auditoría deben ser apropiadas para adaptarse a las 
diferentes circunstancias y deben ser sencillas y concisas, para evitar detalles inútiles y operaciones innecesarias"1.

2. Las técnicas y procedimientos investigadas en el presente estudio serán aplicadas por los profesionales, firmas consultoras, universidades e instituciones educativas en general, en cuanto se refiere a la auditoría académica.

3. Las técnicas y procedimientos aplicables a la auditoría académica son de contexto y de naturaleza distintas a las utilizadas por la auditoría financiera o tradicional, ya sea desde el punto de vista de la metodología y los objetivos que se persiguen.

4. Estetrabajoserviráparapropenderaotorgar un valoragregadoalostrabajosde auditoría académica, por el hecho de asegurar un trabajo profesional de alta calidad.

5. Servirá de base a la actividad académica de las universidades y colegios profesionales, para ser incluidos en sus planes curriculares de estudios.

\section{REFERENCIAS}

Trabajos TécnicosNacionaleseInternacionales. Conferencia Interamericana de Contabilidad,XXIVConferencia.Puntadel Este, Uruguay, 2001.

Trabajos Técnicos Nacionales e Internacionales. XXIII Conferencia Interamericana de Contabilidad. San Juan de Puerto Rico, 1999.
Waintein, Mario y Casal, Armando Miguel. Normas de Auditoría Nacionales e Internacionales. Desar rollo y Gestión. Profesional y Empresarial, Ediciones Errepar, Ciudad Autónoma de Buenos Aires, setiembre de 2000.

Waintein, Mario y Casal, Armando Miguel. "La auditoría Integral en el marco de un mundoglobalizado". XXIIIConferencia Interamericana de Contabilidad, San JuandePuerto Rico, del 02 al 05 de agosto de 1999.

American Accountant Association (citado por Walter Kell y Walliam Boynton. "Auditoría Moderna". Editorial CECSA, México 1999).

Mills, David. Manual de la Auditoría de la Calidad. Ediciones Gestión 2000 S.A. Barcelona, 1997.

ISO 14043. Interpretación de la evaluación del ciclo de vida.

Auditoría de la Cultura Empresarial. Ediciones Díaz de SantosS.A. España, 1992.

Hernández Sampiri, Roberto; Carlos FernándezColladoy PilarBaptista Incio. Metodología de la Investrgación. Mc GrawHill Interamericana S.A., México, 1997. Suárez S., Andrés. La moderna auditoría. Un Análisisconceptualymetodológico, Mc GrawHill de Management, España, 1991.

Pineda, Pilar.Auditoría deFormación. Ediciones Gestión 2000 S.A. Barcelona, 1995.

Elizondo López, Arturo. La Investigación Contable: Significación y Metodología. Ediciones Contables, México, 1992.

Egúsquiza Pereda, Carlos. "Enfoques y objetivos. Hacia una auditoría integrada”. En: Revista Quipukamayoc del Instituto de Investigaciones de Ciencias Financieras y Contables.

\footnotetext{
1 Holmes ArthurW. Auditoría,pnncipiosyprocedimientox. TomoI, Unión Tipográfica Hispano Americana, 2. a ed., 1979.
} 
Hernández V. Jaime. Laaudztoría integral: Un verdadero arquetipo de control empresarial. Documentos y materiales de la Conferencia Interamericana de Contabilidad; Puerto Rico, 1999.

Senge, Peter M. La quinta disciplina: Cómo impulsar el aprendizaje en la organización inteligente, Ediciones Juan Granica S.A. Barcelona.

Eyssautier De La Mora. Metodología en la investigación. Ediciones Contable y Administrativas S.A. - ECASA, México 1991.
Arthur Holmes. Auditoría: Principios y procedimientos. Editorial Hispano Americana - UTHEA, 1979.

InstitutoMexicano de Contadores Públicos. Normasy Procedzmientos de Auditoría, 18. ${ }^{2}$ ed., tomos I y II, 1998.

Instituto Mexicano de Contadores Públicos. Normas Internacional de Auditoría, 3. ${ }^{\text {ra }}$ ed.

J.A-Cashin, P.D.Neuwirth y J.F. Levy. Manual de auditoría. Tomos I, II, y III. Ediciones Centrum Técnicas y Científicas, 1994. 